\title{
3D cardiomyocyte-based biosensor with tissue engineering scaffold and microelectrode array
}

\author{
WEI Xin-wei ${ }^{1}$, GAO Qing², WU Qian', PAN Yu-xiang ${ }^{1}$, QIN Zhen ${ }^{1}$, HU Ning ${ }^{1}$, WANG Ping ${ }^{1, *}$ \\ ${ }_{1}^{1}$ Biosensor National Special Laboratory, Key Laboratory for Biomedical Engineering of Education \\ Ministry, Department of Biomedical Engineering, Zhejiang University, Hangzhou 310027, China; \\ 2 State Key Laboratory of Fluid Power and Mechatronic Systems, Key Laboratory of 3D Printing \\ Process and Equipment of Zhejiang Province, College of Mechanical Engineering, Zhejiang University, \\ Hangzhou 310027, China.
}

\begin{abstract}
:
Polylactic acid (PLA) and polycaprolactone (PCL) were selected as materials to fabricate tissue engineering scaffolds by $3 \mathrm{D}$ printing and electrospinning, which were used to culture cardiomyocytes of neonatal rats. Then the scaffolds with cardiomyocytes were coupled with microelectrode array (MEA) to form a 3D cell-based biosensor, which was used to detect the extracellular field potential (EFP) of cardiomyocytes. The experimental results demonstrated that cardiomyocytes adhered and grew well in scaffolds, and could drive fibers to produce combined beating due to the excitationcontraction coupling. After 48 hours, the beating rate of cardiomyocytes in the scaffolds tended to be stable. The detecting results demonstrated that scaffolds and MEA were coupled well to be a 3D cellbased biosensor system, which could detect the EFP of cardiomyocytes in scaffolds with stable and high-SNR signals, and the EFP amplitude and firing rate are both similar to the signals recorded from traditional 2D culturing method.
\end{abstract}

Key words: 3D cell-based biosensor; tissue engineering scaffold; microelectrode array; cardiomyocyte; extracellular field potential.

\section{Introduction}

Scaffolds, a very important part of tissue engineering, provide an ideal environment for cell attachment, migration and proliferation [1]. At present, there are many technologies for manufacturing tissue engineering scaffolds. Electrospinning, which can continuously produce nanometer or submicron Microfiber, has become the most important method of making tissue engineering scaffolds [2].

Microelectrode array (MEA) is one of the most commonly used cell-based biosensor, which

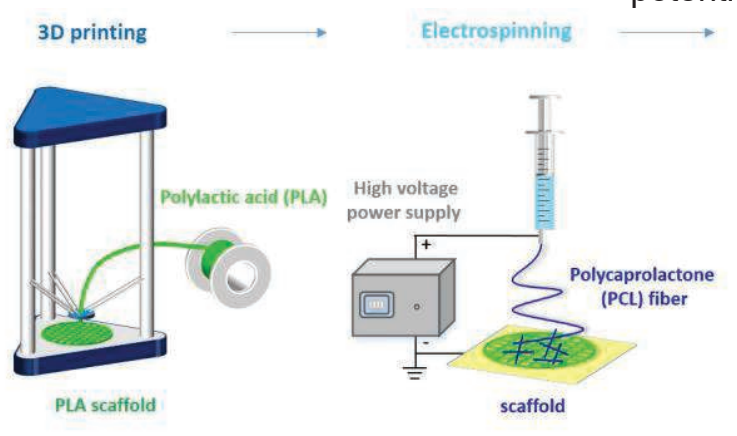

can record extracellular potential signals of Cardiomyocytes or neurons [3]. Traditionally, to build a cell-based biosensor, cells are directly cultured on the sensor chip. However, this simplified method is difficult to reflect true response for lacking of the characteristics of in vivo $3 \mathrm{D}$ tissue [4].

In this study, we combined tissue engineering scaffolds and MEA chip to construct 3D cellbased biosensor, which can form a 3D cell sensing system to mimic the biological in vivo environment and detect extracellular field potential of cardiomyocytes.

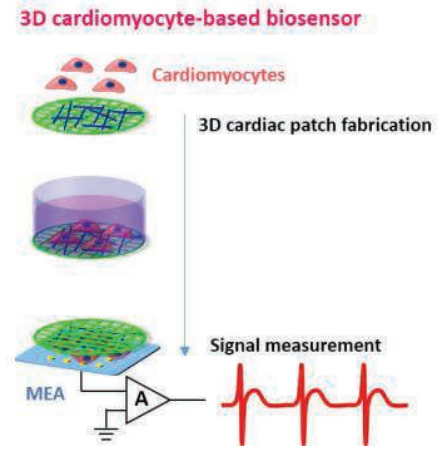

Fig.1. Construction of 3D cardiomyocyte-based biosensor. 


\section{Methods}

Polylactic acid (PLA) and polycaprolactone (PCL) were selected as materials to fabricate tissue engineering scaffolds by $3 \mathrm{D}$ printing and electrospinning. Then the cardiomyocytes of neonatal rats were cultured in scaffolds. The scaffolds with cardiomyocytes were coupled with microelectrode array (MEA) to form a 3D cell-based biosensor, which was used to detect the extracellular field potential (EFP) of cardiomyocytes in scaffolds (see Fig. 1).

\section{Results}

The experimental results demonstrated that cardiomyocytes adhered and grew well in scaffolds, and could drive fibers to produce combined beating due to the excitationcontraction coupling. The beating rate of cardiomyocytes tended to be stable after 48 hours and reached $(34.25 \pm 3.3)$ times/min after 72 hours. After coupling cardiomyocytes scaffold with MEA chip, 3D cardiomyocytebased biosensor could record the different EFP patterns of cardiomyocytes in scaffolds with stable and high-SNR signals (see Fig. 2).

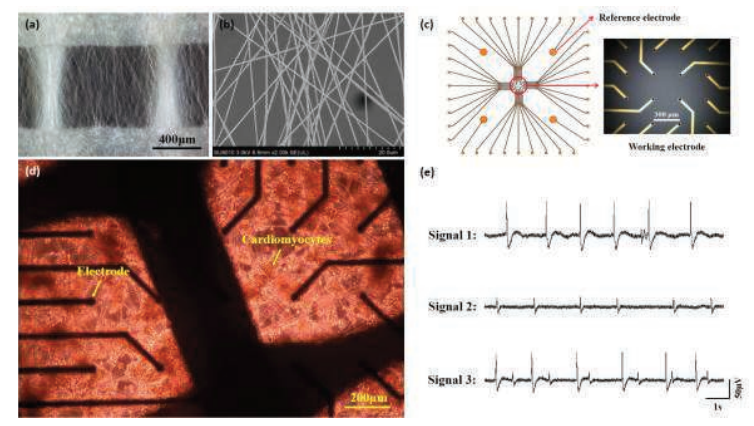

Fig.2. (a)Optical micrograph of scaffold; (b)SEM micrograph of scaffold; (c)MEA sensor chip; (d) Coupling of cardiomyocytes scaffold and MEA chip; (e) Different EFP patterns of cardiomyocytes in scaffold

EFP signal patterns were related to pacemaker and cell status of cardiomyocytes in scaffold. Before cardiomyocytes in scaffold fused fully to form synchronous beating, there may be multiple pacemaker points, so the sensor would record the EFP signals from several pacing points simultaneously. The more active the cardiomyocytes in scaffold behave, the larger EFP signal amplitude and the higher beating rate will be.

\section{Discussion}

EFP signals of cardiomyocytes cultured in planar sensors and tissue engineering scaffolds are recorded respectively (see Fig. 3a). The amplitude and firing rate of EFP signal were statistically analyzed (see Fig. 3b-3c). The EFP signal amplitude of cardiomyocytes in 2D culture enviroment was $(228.9 \pm 6.9) \mu \mathrm{V}$, and the firing rate was $(34.2 \pm 4.6)$ times/min. Similarly, in 3D scaffold culture environment, EFP signal amplitude was $(222.6 \pm 41.9) \mu \mathrm{V}$, and the firing rate was $(33.9 \pm 8.4)$ times $/ \mathrm{min}$. Significant difference analysis was conducted with $t$ test, and it was found that the EFP signals recorded in the two culture environments showed no significant difference in the amplitude $(p>0.05, n=29)$ and firing rate $(p>0.05, n=10)$.

In conclusion, this study provides a new method to construct 3D cell-based biosensor, which could detect the EFP of cardiomyocytes in scaffolds with stable and high-SNR signals. Cells in scaffolds can mimic the in vivo environment and has more physiological significances. This study is expected to be applied in the field of cardiac drug assessment and tissue engineering.

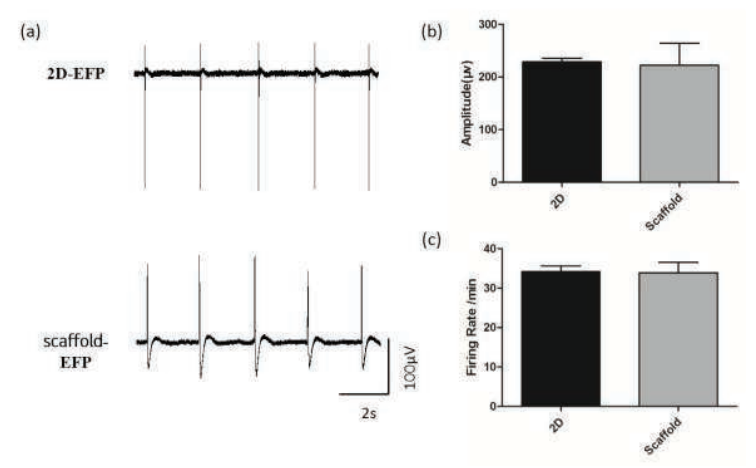

Fig.3. (a) EFP signals of cardiomyocytes cultured in planar sensors and scaffolds; (b) EFP firing rate in two modes of cultivation; (c) EFP amplitude in two modes of cultivation

\section{References}

[1] Sachlos E, Czernuszka J T. Making tissue engineering scaffolds work. Review: the application of solid freeform fabrication technology to the production of tissue engineering scaffolds, European Cells \& Materials, 5(29), 39-40 (2003); doi: 10.22203/ecm.v005a03

[2] Pham Q P, Sharma U, Mikos A G. Electrospinning of polymeric nanofibers for tissue engineering applications: a review, Tissue Engineering, 12(5), 1197-1211 (2006); doi: 10.1089/ten.2006.12.1197

[3] Spira M E, Hai A. Multi-electrode array technologies for neuroscience and cardiology, Nature nanotechnology, 8(2), 83-94 (2013); doi: doi:10.1038/nnano.2012.265

[4] Toh Y C, Zhang C, Zhang J, et al. A novel 3D mammalian cell perfusion-culture system in microfluidic channels , Lab on a Chip, 7(3), 302309 (2007); doi: 10.1039/B614872G 